\title{
ENTOMOLOGÍA MÉDICA
}

\section{Garrapatas (Acari: Ixodidae) prevalentes en perros no migrantes del noroccidente de Bogotá, Colombia}

\author{
M. E. J. Acero',2, O. J. Calixto ${ }^{1}$, A. C. Prieto ${ }^{1}$ \\ 1 Grupo de Patogenicidad Bacteriana, Facultad de \\ Medicina, Universidad Militar Nueva Granada, Bogotá, \\ D.C., Colombia \\ 2 Grupo de Procesamiento de Señales e Imágenes, \\ Facultad de Medicina, Universidad de La Sabana, \\ Bogotá, D.C., Colombia
}

Introducción. En Bogotá, ciudad ubicada en latitud norte $4^{\circ} 035$, longitud oeste $74^{\circ} 4^{\prime}$, con altura $2.640 \mathrm{msnm}$, se documentaron aumentos históricos de temperatura en la primera década de siglo XXI; cerca de la ciudad se ha demostrado la presencia de Amblyomma cajennense, Rhipicephalus sanguineus, y Anocentor nitens en perros y équidos, pero no en perros domiciliados de estancia permanente en la ciudad.

El objetivo del estudio fue determinar la presencia de garrapatas en perros no migrantes del noroccidente de Bogotá.

Materiales y métodos. Se llevó a cabo un estudio descriptivoobservacionalentresclínicasveterinarias particulares durante el 2010; se recolectaron garrapatas de perros domiciliados y cuyos propietarios demostraron estancia permanente en Bogotá. Las garrapatas se recolectaron de forma manual y se fijaron en formol al $10 \%$. En el Laboratorio de Microbiología de la Facultad de Medicina de la Universidad Militar Nueva Granada, se examinaron y se identificaron usando claves taxonómicas pictóricas y de nomenclatura.

Resultados. Un total de 799 perros cumplieron el criterio de inclusión; se hallaron 34 garrapatas (una por animal) lo que constituyó una tasa de infestación de 4,25\%. Las garrapatas recolectadas fueron Acari: Ixodidae, y 22 se clasificándose como R. sanguineus, 11 como R. (Boophilus) microplus, y una $A$. maculantum.

Conclusiones. Este es el primer estudio que identifica garrapatas Acari: Ixodidae en perros domiciliados dentro de Bogotá. Empiezan a ser necesarios cambios de los paradigmas que orientan las políticas de salud pública; el riesgo de la población a zoonosis trasmitidas por garrapatas $R$. sanguineus, R. (Boophilus) microplus $y$
A. maculatum no se conoce. Es necesaria la realización de futuros estudios que permitan establecer la importancia de estos hallazgos.

\section{Identificación de una mutación puntual relacionada con la resistencia a organofosforados en una carboxilesterasa de Rhipicephalus microplus}

Edgar Díaz-Rivera, Gustavo Adolfo Vallejo

Laboratorio de Investigaciones en Parasitología

Tropical, Universidad del Tolima, Ibagué, Colombia

Introducción. Algunos estudios han identificado alteraciones moleculares generadoras de resistencia a acaricidas en garrapatas, problema bastante difundido en la ganadería mundial. Uno de los polimorfismos se encuentra en una carboxilesterasa que inhibe la acción de los organofosforados sobre Rhipicephalus microplus.

El objetivo de investigación fue determinar si en Colombia, las poblaciones de campo de la garrapata R. microplus resistentes a organofosforados poseen la mutación puntual reportada en garrapatas de otros países.

Materiales y métodos. Veintiocho teleoginas de $R$. microplus recolectadas en campo fueron sometidas a una prueba de inmersión para detección de resistencia, sumergiéndolas tres minutos en dilución comercial de un organofosforado y manteniéndolas en incubación durante 18 días, para obtener garrapatas sensibles, moderadamente resistentes y resistentes según la supervivencia y el volumen de oviposición. Se extrajo el ADN de cada garrapata empleando el método fenol-cloroformo, previa digestión enzimática con proteinasa K. Con cada muestra se realizó un procedimiento de PCR para generar un fragmento de $372 \mathrm{pb}$ correspondiente a la carboxilesterasa Est9 y una prueba de RFLP con endonucleasa $E c o R I$ para diferenciar genotipos, separándolos por electroforesis en gel agarosa al $1,5 \%$. Para analizar los datos se empleo la prueba exacta de comparación de proporciones con intervalo de confianza del $95 \%$.

Resultados. Las garrapatas con algún nivel de resistencia generaron dos segmentos a partir del fragmento de $372 \mathrm{pb}$ (22/26), demostrando 
presencia de la mutación puntual, mientras las sensibles no presentaron división del fragmento $(0 / 2)$. Las diferencias fueron estadísticamente significativas $(p<0,05)$ con relación directa entre el genotipo y el fenotipo con un nivel de confianza de $\mathrm{p}=0,039$.

Conclusiones. Se demostró la presencia de una mutación puntual en la carboxilesterasa Est9 de garrapatas $R$. microplus resistentes a organofosforados, siendo éste el primer reporte en Colombia.

$$
\bullet \bullet \bullet
$$

\section{Evaluación de péptidos recombinantes derivados del péptido SBm7462 ${ }^{\circledR}$, respuesta inmune y alteraciones histológicas del intestino medio de Rhipicephalus (Boophilus) microplus}

Gabriel A. Tafur ${ }^{1}$, Joaquín H. Patarroyo', Sidimar Sossai ${ }^{1}$, Marlene I. Vargas ${ }^{1}$, Karlos E. Kalks ${ }^{1}$, Adriana M. Henao', Leandro Silva de Araujo', Cintia Fidelis', Byron A. Hernández ${ }^{1}$, Ronny Francisco de Souza ${ }^{2}$

1 Laboratório de Biologia e Controle de Hematozoários e Vetores, Instituto de Biotecnologia Aplicada à Agropecuária, Departamento de Medicina Veterinária, Universidade Federal de Viçosa, Viçosa, Minas Gerais, Brasil

${ }^{2}$ Laboratório de Infectologia Animal Instituto de Biotecnologia Aplicada à Agropecuária - BIOAGRO, Universidade Federal de Viçosa, Viçosa, Minas Gerais, Brasil

Introducción. Los perjuicios generados por Rhipicephalus microplus tienen implicaciones en la salud pública y en la producción animal. El control por vacunas es una alternativa viable que no genera garrapatas resistentes y ofrece inocuidad tanto a los productos de origen animal como al medio ambiente.

El objetivo de este estudio fue evaluar la respuesta inmune en ganglios linfáticos bovinos y las alteraciones histológicas en hembras de $R$. microplusinducidas pordos péptidos recombinantes derivados del péptido SBm7462 ${ }^{\circledR}$.

Materiales y métodos. Se diseñaron y sintetizaron los genes seq1 (150 aminoácidos) y seq4 (45 aminoácidos) para la expresión proteica en la levadura Pichia pastoris, cepa Km71; los péptidos expresados se caracterizaron por SDSPAGE y Western blot. Dos mg de cada péptido más $1,5 \mathrm{mg}$ de saponina, fueron inoculados, por vía subcutánea, tres veces a intervalos de 30 días en 10 becerros Bos taurus divididos en dos grupos (A seq1 y B seq4). Para el desafío, se colocaron 4.500 larvas de $R$. microplus por animal,
28 días después de la última inmunización. Los ganglios linfáticos recolectados en diferentes tiempos, se procesaron para hematoxilina $y$ eosina y peroxidasa-antiperoxidasa (PAP). De las garrapatas naturalmente desprendidas, se selecionaron aleatoriamente 10 de cada grupo y las asas intestinales se procesaron para histología, y se tiñeron con hematoxilina y eosina, Giemsa lento e inmunoperoxidasa indirecta.

Resultados. Se caracterizó una respuesta dependiente de linfocitos $T$, con formación de centros germinales que se diferenciaron en zona clara y oscura en los animales del grupo B, así como una fuerte marcación de células positivas para PAP en las regiones paracorticales y en los cordones medulares. Además, las teleoginas de $R$. microplus que parasitaron a los animales inmunizados $r B m s e q 4$, presentaron alteraciones en el epitelio intestinal.

Conclusiones. Existe una respuesta inmune protectora contra $R$. microplus al utilizar el péptido rBmseq4.

\section{Diversidad de mosquitos (Diptera: Culicidae) en el área de influencia de la hidroeléctrica del río Sogamoso, Santander}

Gabriel Jaime Parra-Henao, Erika Patricia Alarcón, Daniel Delgado, Laura Bastidas

Instituto Colombiano de Medicina Tropical, Universidad CES, Sabaneta, Colombia

Introducción. La familia Culicidae (Diptera: Culicidae) incluye géneros y especies de gran importancia como vectores de enfermedades como malaria, dengue, fiebre amarilla, virus de la encefalitis equina, virus del oeste del Nilo y otros arbovirus.

El objetivo del estudio fue determinar la diversidad de mosquitos (Diptera: Culicidae) en el área de influencia de la hidroeléctrica del río Sogamoso en el departamento de Santander.

Materiales y métodos. El estudio se llevó a cabo entre noviembre de 2009 y agosto de 2010. Se realizaron seis visitas bimestrales para recolectar mosquitos culícidos, en el intradomicilio, peridomicilio y extradomicilio de viviendas pertenecientes a las veredas ubicadas en la zona de influencia del embalse (municipios de Betulia, Girón, Los Santos, San Vicente de Chucurí, Lebrija y Zapatoca). Para la recolección de los mosquitos adultos se utilizaron trampas CDC (18:00 a 06:00 horas) y trampas Shannon (18:00 a 22:00 horas). También se recolectaron mosquitos en reposo 
en las horas del día. Los estadios inmaduros se muestrearon en criaderos naturales y artificiales.

Resultados. Se obtuvieron 2.277 mosquitos culícidos adultos y 1.629 inmaduros (larvas y pupas) de 12 géneros y 30 especies. Los géneros Culex, Aedes y Psorophora tienen amplia dispersión en la zona de estudio. El género Anopheles es de distribución restringida.

Conclusiones. Las especies Anopheles nuñeztovari, An. pseudopunctipennis, Aedes aegypti, Haemagogus equinuus, Psorophora confinnis, $P$ s. ferox, Culex quinquefasciatus, Cx. coronator $y$ Coquillettidia venezuelensis halladas en la zona de estudio, alertan sobre la posibilidad de transmisión de diferentes enfermedades transmitidas por vectores.

\section{Inclusión de la entomología médica en el plan de estudios de medicina de la Universidad Libre, Seccional Cali}

Greysy Dayana García, Marcia Liseth Moreno, Daniela Larrahondo, Licenia Frades

Programa de Medicina, Universidad Libre, Cali, Colombia

Introducción. El programa de Medicina de la Universidad Libre de Cali tiene una trayectoria de 36 años, desde el 2006 se encuentra con acreditación de alta calidad. Una de las recomendaciones dadas por el Comité Nacional de Acreditación fue ampliar el componente de electivas en el programa y es aquí donde se propone incluir la entomología médica como fortalecimiento al futuro profesional de Medicina, teniendo como base que el grupo de enfermedades transmitidas por vectores entomológicos tienen un valor significativo en la salud pública de nuestro territorio.

Materiales y métodos. La inclusión de la electiva siguió todos los conductos regulares correspondientes al nivel académico de un programa profesional.

Para el desarrollo del mismo se realizaron las siguientes estrategias metodológicas: exposición magistral, presentación y análisis de casos, talleres y laboratorios teórico-prácticos y un trabajo final referente a los vectores entomológicos.

Resultados. Durante el desarrollo de la asignatura, se han realizado actividades académicas con miras a resaltar la importancia de la entomología médica. De las cuales se destacan: la presentación de casos clínicos de dengue y malaria por los estudiantes en su rotación por el Hospital Infantil Club Noel; la elaboración de material educativo y explicación a la comunidad afectada; la captura de artrópodos y la organización de la colección; la participación en eventos académicos y en brigadas de salud organizadas por el programa de Medicina en el que la formación en entomología medica es muy significativa.

Conclusiones. La entomología médica dentro del plan de estudios de Medicina es una fortaleza para el futuro profesional porque con ella aprende las estrategias de manejo de los vectores entomológicos de gran significancia en la salud pública. Además, puede ser ofrecida a diferentes programas de formación profesional.

\section{Miasis sinusal y traqueo-pulmonar: reporte de un caso y revisión de la literatura}

Héctor Julio Meléndez ${ }^{1}$, Yenny Tamayo-Cáceres², Yeny

Tello-Olarte ${ }^{2}$, Roger Tarazona², Francisco Vargas ${ }^{2}$

1 Hospital Universitario de Santander, Universidad Industrial de Santander, Bucaramanga, Colombia Universidad Industrial de Santander, Bucaramanga, Colombia

Introducción. La miasis es una enfermedad causada por infestación de larvas en tejidos vivos o muertos del huésped; se clasifica entomológicamente o según el trofismo por los tejidos.

Caso clínico. Se reporta un caso inusual de miasis traqueo-pulmonar y sinusal en un hombre de 65 años con mala higiene y abandono social, dedicado a la cría de animales de campo; ingresó a un hospital local con epistaxis y cifras elevadas de presión arterial. Se manejó sintomáticamente y se le dio de alta con tapón nasal; al día siguiente regresó con dificultad respiratoria, se le retiró el tapón nasal y se evidenció la salida de larvas.

Fue llevado a cirugía por el Servicio de Otorrinolaringología, y se le extrajeron, aproximadamente, 150 larvas de la cavidad nasal, etmoidal y retrofaríngea; posterior al procedimiento requirió hospitalización en la unidad de cuidados intensivos por descompensación hemodinámica y síndrome grave de dificultad respiratoria.

En las imágenes diagnósticas y en la broncofibroscopia se observaron irregularidades de aspecto polipoide en la superficie de la mucosa traqueal, la clínica del paciente y las ayudas diagnósticas confirmaron la migración de las larvas a las vía aérea inferior. Se dio manejo con ivermectina entérica y antibióticos profilácticos, como está reportado en la literatura, y se obtuvo buena respuesta al tratamiento. 
Conclusiones. En este caso se planteó la posibilidad de miasis de la vía aérea inferior en un paciente que presentaba múltiples factores de riesgo, inicio con miasis nasal y progresión con lesión pulmonar seria que evolucionó a un síndrome de dificultad respiratoria y estenosis desde la glotis hasta la carina.

En la literatura revisada, se encontraron descritos sólo cuatro casos de miasis de localización traqueopulmonar. Con este reporte se busca resaltar la importancia de indagar en los antecedentes socieconómicos y recordar los signos y síntomas de las miasis de cavidades y de la vía aérea.

\section{Nichos ecológicos y propensión a la infección de los vectores de la esquistosomiasis en las cuencas del río Paraná y Uruguay en la provincia de Corrientes, Argentina}

María Josefa F. Rea, C. Edgardo Borda, Osvaldo

Benítez, Luis Armando Mosqueda

Centro Nacional de Parasitología y Enfermedades

Tropicales, Facultad de Medicina, Universidad Nacional del Nordeste, Corrientes, Argentina

Introducción. En Argentina se encuentran diferentes especies de moluscos, entre ellos se destacan los caracoles del género Biomphalaria, transmisores de Schistosoma mansoni. La esquistosomiasis se ha expandido a los estados del sudeste del Brasil, limítrofes con la provincia de Corrientes. La determinación de la distribución de las especies de Biomphalara propensas a la infección brinda información de las posibles áreas donde esta enfermedad podría aparecer.

El objetivo de este estudio fue caracterizar la distribución de las especies de Biomphalaria de la provincia de Corrientes y verificar la propensión a la infección por S. mansoni.

Materiales y métodos. La provincia de Corrientes está ubicada en la región Mesopotámica del nordeste de Argentina, con clima subtropical húmedo.

Los moluscos fueron recolectados en hábitats naturales (arroyos, ríos, pantanos, zanjas) y artificiales (canales, represas) de 11 departamentos de la provincia, cinco de la cuenca del río Paraná y seis de la del Uruguay. Ambos pertenecen a la cuenca del río de la Plata.

Grupos de 100 caracoles $F_{1}$ de Biomphalara tenagophila recolectados en 17 biótopos fueron expuestos individualmente con 10 miracidios de la cepa $\mathrm{SJ}_{2}$ de São Paulo, Brasil.
Resultados. Se identificaron dos especies de huéspedes intermediarios naturales en Brasil: Biomphalaria tenagophila y $B$. straminea, un potencial vector como $B$. peregrina, además de $B$. orbigny. Biomphalara tenagophila fue la especie más frecuente (76\%). Todos fueron negativos para S. mansoni.

En la cuenca del Paraná los biótopos más frecuente para $B$. tenagophila fueron los arroyos y los caracoles de cuatro departamentos fueron propensos a la infección por $S$. mansoni.

En la cuenca del Uruguay, se obtuvo B. tenagophila de 16 biótopos de cinco departamentos, $62 \%$ eran artificiales, y los caracoles de tres departamentos eran propensos a la infección.

Conclusiones. Se confirma la amplia distribución de Biomphalaria tenagophila en diversos biótopos de esta región, así como la propensos a la infección de muchas poblaciones a $S$. mansoni.

\section{Siphonaptera (Pulicidae) en viviendas de Bogotá, Colombia}

Maritza Torres ${ }^{1}$, Elizabeth García², Evelyne Halpert², Elizabeth Borrero², Pablo Chaparro², Milcíades lbáñez², Jorge Molina ${ }^{1}$

${ }^{1}$ Centro de Investigaciones en Microbiología y

Parasitología Tropical, Universidad de los Andes, Bogotá, D.C., Colombia

2 Fundación Santa Fe de Bogotá, Bogotá, D.C., Colombia

Introducción. Las pulgas (Siphonaptera: Pulicidae) son ectoparásitos que por sus picaduras pueden causar anemias y reacciones alérgicas, o transmitir agentes infecciosos a animales domésticos y humanos. La presencia de pulgas en las viviendas es conocida en varias ciudades de Colombia pero no se han realizado hasta el momento trabajos que cuantifiquen su presencia en los domicilios. Con este trabajo se busca determinar la distribución y las especies de pulgas presentes en Bogotá.

Materiales y métodos. Se colocaron trampas adhesivas sin luz (control) y con estímulos lumínicos (azul, 454 \pm 30 , y verde, $520 \pm 30 \mathrm{~nm}$ ) en 280 viviendas pertenecientes a las 19 localidades de Bogotá. Cada trampa tenía un área de muestreo de $245,44 \mathrm{~cm}^{2}$ impregnada en aceite de ricino. Se colocaron pares de trampas (control y tratamiento) a $10 \mathrm{~cm}$ de distancia entre ellas en diferentes habitaciones de las viviendas. Luego de tres días de muestreo, las trampas se recuperaron y se revisaron para detectar la presencia de pulgas. Las especies se aclararon con $\mathrm{KOH}$ al $10 \%$ por 24 horas y se deshidrataron con pasos sucesivos de alcohol para su identificación. 
Resultados. Once de las 19 localidades de Bogotá registraron presencia de pulgas. De las 280 viviendas muestreadas, 30 resultaron positivas $(10,7 \%)$. En total, se recolectaron 61 pulgas; Pulex irritans (19 machos y 18 hembras) fue la especie más abundante, seguida por Ctenocephalides felis (17 machos y 6 hembras) y Ctenocephalides canis (1 hembra). Con las trampas de luz azul se capturaron significativamente más pulgas que con las trampas sin luz o con luz verde.

Conclusión. Pulex irritans y C. felis son dos de las especies de pulgas más frecuentemente reportadas en condiciones urbanas con variaciones en abundancia en diferentes países. Nuestras capturas con trampas de luz demuestran su utilidad para los estudios epidemiológicos con pulgas.

Financiado por Colciencias contrato 571-2008.

$$
\text { - } \bullet
$$

\section{Características de los cultivos celulares derivados de tejidos embrionarios de Sarconesiopsis magellanica (Diptera: Calliphoridae)}

Mónica Cruz, Felio Bello

Laboratorio de Entomología Médica y Forense, Universidad del Rosario, Bogotá, D.C., Colombia

Introducción. Sarconesiopsis magellanica es una mosca necrófaga de interés médico-forense, debido a su uso en la determinación del intervalo post mórtem y, también, potencialmente, podría tener aplicación en terapia larvaria. No se conocen cultivos celulares derivados de esta especie. Las líneas celulares de insectos se emplean en estudios biomédicos y biotecnológicos.

El objetivo principal de este estudio fue obtener y caracterizar cultivos celulares primarios derivados de tejido embrionario de $S$. magellanica.

Materiales y métodos. Se realizaron explantes de huevos con embrión y larvas neonatas en cuatro medios de cultivo diferentes: MM/VP12, L15 y Grace/L15 y Schneider, con suplemento de suero fetal bovino al $20 \%$ y una mezcla de antibióticos y antimicóticos al 1\% con pH de 6,8. La disgregación de las células se realizó mediante la homogeneización de tejidos y tripsina al 1\%. La temperatura de incubación de los cultivos primarios fue de $28{ }^{\circ} \mathrm{C}$, sin atmósfera de $\mathrm{CO}_{2}$. Mediante la observación microscópica directa y pretratamientos se determinaron las características morfológicas de las células y del cariotipo, respectivamente.

Resultados. Los medios que brindaron las mejores condiciones para la adhesión y proliferación celular fueron L-15 y Grace/L15. En el patrón de crecimiento de los cultivos primarios se encontró la presencia de vesículas adheridas a fragmentos de embriones y también en suspensión, las cuales contribuyeron a la dispersión de las nuevas células. La morfología celular predominante en la monocapa correspondió a fibroblastoides y, en menor proporción, a epitelioides. Además, en las monocapas confluentes las células fibroblastoides tenían una apariencia parecida a las nerviosas. También, se registraron en la monocapa movimientos contráctiles similares a los realizados por células musculares. El número cromosómico diploide de las células fue de 12 .

Conclusiones. Los cultivos celulares obtenidos, bajo las características morfológicas y citogenéticas propias de esta especie, constituyen un sustrato potencialmente útil para futuros estudios de propensión a infecciones con arbovirus y parásitos.

\section{Distribución urbana de las especies del género Haemagogus en dos departamentos de la Región Caribe colombiana}

Ronald Maestre-Serrano', Suljey Cochero², Betsy BelloNovoa $^{3}$, Cristina Ferro ${ }^{3}$

1 Grupo de Investigación en Enfermedades Tropicales y Biomédicas del Atlántico, Secretaria de Salud del Atlántico, Barranquilla, Colombia

2 Laboratorio de Entomología, Dasssalud-Sucre, Grupo de Investigaciones Biomédicas, Universidad de Sucre, Sincelejo, Colombia

3 Laboratorio de Entomología, Instituto Nacional de Salud, Bogotá, D.C., Colombia

Introducción. Los mosquitos del género Haemagogus son importantes en salud pública porque algunas de sus especies participan como vectores en el ciclo selvático del virus de la fiebre amarilla. Para Colombia se han identificado como vectores principales las especies $H$. janthinomys y $H$. equinus El objetivo del presente trabajo consistió en actualizar el registro y la distribución de especies del género Haemagogus, en las áreas urbana y periurbanas de los departamentos de Atlántico y Sucre en la región Caribe colombiana.

Materiales y métodos. Se revisó la colección entomológica de referencia para especies del género Haemagogus constituida de larvas de tercer y cuarto estadio, recolectadas en larvitrampas, y adultos obtenidos a partir de series entomológicas. Para la determinación de las especies se utilizaron los caracteres morfológicos y las claves taxonómicas de Arnell, 1973. 
Para conservar las cerdas de las larvas, como caracteres diagnósticos, los especímenes se preservaron en alcohol al $70 \%$ y luego se miraron al estereomicroscopio sin aclarar, sobre una gota de agua. También se realizaron montajes en bálsamo de Canadá de la terminalia de los machos y de algunas larvas para los caracteres morfológicos específicos.

Resultados. Se confirma la presencia en el departamento del Atlántico de $H$. anastasionis y $H$. celeste en el Batallón Vergara y Velazco del municipio de Malambo, el aeropuerto Ernesto Cortissoz, la terminal de transporte, la central de abastos y la base aeronaval del municipio de Soledad, y $H$. equinus, en la terminal de transportes y en la central de abastos del municipio de Soledad.

Para el departamento de Sucre se confirma $H$. equinus, $H$. anastasionis y $H$. celeste en el aeropuerto del municipio de Santiago de Tolú y constituyen nuevos registros de este género para el departamento.

Conclusiones. Se aumentó el número de registros de tres especies del género Haemagogus en la región Caribe colombiana. Se confirmó la adaptación de las especies $H$. anastasionis, $H$. celeste y $H$. equinus a recipientes artificiales en el área urbana de ambos departamentos.

\section{Características del ciclo biológico y tablas de vida de Sarconesiopsis magellanica (Diptera: Calliphoridae), bajo condiciones de laboratorio}

Tatiana Pinilla, Felio Bello

Laboratorio de Entomología Médica y Forense, Universidad del Rosario, Bogotá, D.C., Colombia

Introducción. Sarconesiopsis magellanica es una mosca de importancia médica y forense, empleada para establecer el intervalo post mórtem. Sin embargo, no se conoce el ciclo de vida ni los parámetros biológicos de esta especie.

El objetivo del estudio fue determinar las características del ciclo biológico del insecto y la construcción de tablas de vida, bajo condiciones de laboratorio, empleando tres dietas diferentes.

Materiales y métodos. Se recolectaron especímenes adultos en varios sitios de la sabana de Bogotá y se llevaron al laboratorio, bajo condiciones físicas y ambientales previamente establecidas y controladas $\left(24{ }^{\circ} \mathrm{C}, 60 \%\right.$ de humedad relativa, fotoperiodo de 12 horas de luz). El sustrato principal de cada una de las tres dietas utilizadas fue: hígado de cerdo, hígado en polvo y huevo-leche en polvo. Se registraron datos de la duración del ciclo de vida y la longevidad de los adultos, a partir de tablas de vida, tamaño de los especímenes y supervivencia. Resultados. La duración del ciclo biológico desde huevo hasta adulto fue de 43,5 días para la dieta de hígado en polvo, 50.5 días para la dieta huevoleche en polvo y 59,5 días para la dieta de hígado de cerdo. El estadio de pupa presentó la diferencia más notable en las tres dietas, siendo la de huevoleche en polvo la de mayor duración (17 días). El análisis de las tablas de vida mostró que los adultos hembras fueron más longevos que los machos, en tanto que la supervivencia más alta se obtuvo en la dieta de hígado de cerdo (85,19\%). Los individuos de esta última dieta fueron los de mayor tamaño. Las curvas de supervivencia representaron una población con un número constante de moscas que murieron en cada intervalo de edad.

Conclusiones. Las dietas utilizadas representaron sustratos adecuados para la cría de mosca en condiciones de laboratorio, posibilitando así su mantenimiento como una colonia estable, la cual ha suministrado el material larvario para ensayos aplicados de terapia con larvas.

\section{Prevalencia de escabiosis en niños de 5 a 10 años de las escuelas públicas de la comuna 1}

Vivian Villalba, Jennifer Carvajal, Gener Rada, Gustavo Ayarza

Universidad del Magdalena, Santa Marta, Colombia

Introducción. La escabiosis es una infección causada por Sarcoptes scabiei, con alta tasa de contagio entre humanos y difícil de erradicar, siendo considerada dentro del grupo de las enfermedades desatendidas.

El objetivo fue conocer la prevalencia de la escabiosis e identificar los factores que influyen para facilitar su presencia y distribución en la población de estudio.

Materiales y métodos. Se realizó un muestreo estratificado, de aisgnación aleatoria, de 405 niños de escuelas de la comuna 1 con edades entre 5 y 10 años, a quienes se les aplicó una encuesta a través de sus padres. Los niños con síntomas sugestivos de escabiosis fueron citados para prueba de raspado y visualización del ácaro con $\mathrm{KOH}$ en el microscopio óptico. A todos los padres o tutores legales de los niños participantes, se les solicitó la firma del consentimiento informado, una vez se hubo explicado el procedimiento. Los datos 
se almacenaron en el programa SPSS $\circledast$, versión 15. Las variables se comparacon con la prueba de ji al cuadrado y análisis de regresión. Se consideró significativa toda $p$ menor de 0,05.

Resultados. Se encuestaron 405 niños de las escuelas de la comuna 1. La media de edad fue de 11,26 años. La prevalencia de escabiosis fue de $9,6 \%$. La prevalencia más alta estuvo en los niños entre 9 y 10 años (2,2\%). El 53,8\% de los afectados reside en el estrato 1 . El prurito estuvo presente en
$23,2 \%$ de los afectados. Algunos de los factores con posible influencia para la escabiosis fueron: el hacinamiento, los antecedentes dermatológicos y la presencia o ausencia de servicios públicos $(p<0,05)$.

Conclusión. La infección por escabiosis es importante en nuestro medio y tiene relación con las condiciones socioeconómicas. 